\title{
Caregiver empowerment program based on the adaptation model increase stroke family caregiver outcome
}

\author{
Kelana Kusuma Dharma ${ }^{a}$, Dedi Damhudia, Nelly Yardes ${ }^{b}$, Suhana Haeriyantob \\ ${ }^{a}$ School of Nursing, Poltekkes Kemenkes Pontianak, Pontianak 78244, Indonesia \\ ${ }^{b}$ School of Nursing, Poltekkes Jakarta III, Pondok Melati, Bekasi, West Java 17415, Indonesia
}

Received: 22 December 2020; Accepted: 9 February 2021; Published: 20 December 2021

\begin{abstract}
Objective: Post-stroke disability and psychosocial disorders cause burdens for the families of stroke patients, including physical and financial burdens. The physical and psychological health of family caregivers determines the quality of care they provide to patients. The purpose of this study was to identify the effectiveness of the Caregiver Empowerment Program Based on the Adaptation Model (CEP-BAM) in increasing the family caregiver's coping ability against various problems and reducing their burden while caring for the stroke patient.

Methods: This research was a quasi-experimental study with pre- and post-test control group design. The intervention group received CEP-BAM, while the control group received a conventional intervention in the form of a discharge planning program for family caregivers in the hospital. The samples were caregivers who care for and facilitate the recovery of stroke patients during their convalescence at home. We selected the samples from the population using the stratified random sampling method. The number of samples completed in the study was 40 in the intervention group and 40 in the control group. Measurement of outcome variables (coping and caregiver burden) was carried out 4 times including pre-test before the intervention, post-test 1 at 4 months after the intervention, post-test 2 at 5 months after the intervention, and post-test 3 at 6 months after the intervention.

Results: There were significant differences in caregiver's coping $(P=0.016)$ and caregiver's burden $(P=0.009)$ in measurements between the two groups.

Conclusions: The CEP-BAM interventions were effective in increasing adaptive coping strategies and reducing the burden of caregivers 4 months and continuing 6 months after the intervention.
\end{abstract}

Keywords: E family caregivers • empowerment • coping behavior $\bullet$ stroke rehabilitation

(c) Shanxi Medical Periodical Press.

\section{Introduction}

The financial burden due to an increase in the prevalence and impact of non-communicable diseases is quite large. One such non-communicable disease whose prevalence continues to increase and which causes a large financial burden is stroke. The disease greatly contributes to increasing the burden of disease

How to cite this article: Dharma KK, Damhudi D, Yardes N, Haeriyanto S. Caregiver empowerment program based on the adaptation model increase stroke family caregiver outcome. Front Nurs. 2021;4:419-428.

\footnotetext{
* Corresponding author.

E-mail:kelana_kusuma@poltekkes-pontianak.ac.id; kelana_dharma@yahoo.com (K.-K. Dharma).
} 
for patients, their families, and the national health system. Patients who survive a stroke mostly experience sequelae due to central nervous system damage such as hemiparesis or hemiplegia, aphasia, dysphagia, cognitive impairment, or memory disorders that cause post-stroke disabilities. Some patients also experience psycho-social disorders such as anxiety and depression after stroke. Disability and psycho-social disorders experienced by patients cause burdens for their family including physical burdens, loss of productivity at work, and financial burden.

Post-stroke disability is related to family burden in caring for patients. The high burden of caring for patients causes various psycho-social problems in caregivers such as anxiety and depression. A systematic review and meta-analysis showed that stroke caregivers experience depression in as much as $40.2 \%$ (95\% confidence interval, $30.1 \%-51.1 \%$ ) of patients, and anxiety in $21.4 \%$ (95\% confidence interval, $11.6 \%-35.9 \%) .{ }^{1}$ Other research on stress levels experienced by caregivers in caring for stroke patients reveals that $65 \%$ of caregivers stated that they have a moderate to a severe level of stress, $81 \%$ leave work to treat the patient, more than half of them experience sleep disorders and physical burdens, and $75 \%$ have said they experience psychological instability and financial burden. ${ }^{2}$ Another study showed that $43.9 \%$ of caregivers had anxiety symptoms, $26.5 \%$ had mild to moderate depression symptoms, and $27.4 \%$ had severe depression symptoms. The length of time taken to treat a patient and the cost of care have an impact on anxiety and depression in the caregivers. ${ }^{3}$ There is a significant negative relationship between the burden of caring for stroke patients and the family's quality of life. The higher the burden, the less the quality of life of the family caregiver. ${ }^{4}$ Based on this, health interventions are needed for caregivers of poststroke patients.

The family caregiver plays an important role in the recovery of stroke patients. The caregiver's role includes helping patients meet their basic needs, guiding patients through the process of rehabilitation and adaptation at home, and providing psychological and social support. The physical and psychological health of caregivers determines the quality of care they provide to patients. Furthermore, the quality of care and assistance provided by the caregiver largely determines the quality of life of patients. Caregivers who do not feel the excessive burden in caring for patients will be able to provide better care and assistance, which will have an impact on patients' outcomes. Research shows that adequate physical abilities of the patient are associated with low caregiver burden. ${ }^{5}$ Based on this, it is necessary to empower the family caregiver of poststroke patients with measures that are not only focused on improving the quality of life of patients but also pay attention to the physical and psychological health of the caregiver. The nurses must provide stroke home care guidelines, psychological counseling, and social support to caregivers to improve health outcomes and reduce their burden. ${ }^{3}$

Researchers have developed stroke caregiver intervention aimed at improving caregiver's health outcomes. A study in Thailand proved that the community-based program for family caregivers increased caregiver knowledge and skills, increased the functional capacity of stroke survivors, and reduced the risk of post-stroke complications. ${ }^{6}$ A strength-oriented psychoeducational program improved caregiver outcomes, including competence in caring for stroke patients, problem-solving, and social support satisfaction. In addition, this intervention also improves family function and reduces the burden on the caregiver. ${ }^{7}$ The family centered empowerment program increases the ability of post-stroke patients to perform daily living activities 2 months after the intervention and reduces the family burden 2 weeks after the intervention. ${ }^{8}$ The family involvement and patient-tailored health management program carried out on 19 patients and their caregivers for 12 weeks shows that this intervention increases the patient's functional ability and satisfaction, but needs further research because of the limited sample size. ${ }^{9}$ Some of the above studies have not yet developed an intervention to empower caregivers in caring for patients after stroke, which aims to increase their ability to adapt and reduce their burden in caring for patients.

The present authors in 2018 developed a caregiver empowerment program based on the adaptation model (CEP-BAM), an intervention that educates and trains caregivers to be able to play an effective role in caring for and facilitating patients through the post-stroke adaptation process at home. Researchers provide caregivers with information about stroke and prevention of recurrent stroke, train them in helping patients adapt after stroke, and teach them how to maintain a healthy psychological condition while caring for patients. This intervention can increase the functional capacity and quality of life of patients 6 months after stroke. ${ }^{10}$ Further studies are required to ascertain whether this intervention can improve coping strategies and reduce the burden of caregivers in caring for stroke patients. These two variables are important factors that influence the ability of caregivers to provide post-stroke care at home. This study aims to determine the effectiveness of CEP-BAM interventions in increasing the caregiver's coping ability against various problems and reducing the caregiver's burden while caring for patients. 


\section{Method}

\subsection{Research design}

This research is a quasi-experimental study with preand post-test control group design. We divided the sample into 2 groups: the intervention and control groups. Measurement of outcome variables in both groups was conducted 4 times including pre-test before the intervention, post-test 1 at 4 months after the intervention, post-test 2 at 5 months after the intervention, and post-test 3 at 6 months after the intervention.

\subsection{Sample}

The samples in this study were caregivers who care for and facilitate the recovery of patients during their convalescence at home. Eligibility criteria for the caregivers include the patient's nuclear family (husband/ wife, parents, siblings, son/daughter), the most dominant role in caring for patients, aged 20-60 years, and caring for stroke patients in the mild to partial care category. We select the sample from a population of family caregivers who treated stroke patients post-discharge from 2 regional government general hospitals in West Kalimantan Province and 2 regional government general hospitals in Jakarta. Population data were obtained from medical records at the four hospitals. We selected the samples from the population using the stratified random sampling method. We divided the selected samples into intervention and control groups by matching hospitals. The sample of the intervention group was comprised of caregivers caring for post-discharge patients from 1 hospital in West Kalimantan and 1 hospital in Jakarta, as well as a control group, and was taken from 1 hospital in each province. The sample size in this study was calculated using the formula of the sample size for testing the hypotheses of the difference means between two independent groups as follows:

$$
\mathbf{n} 1=\mathbf{n} 2=\frac{2 \sigma^{2}\left(\mathbf{z}_{1-\alpha / 2}+\mathbf{z}_{1-\beta}\right)^{2}}{\left(\mu_{1}-\mu_{2}\right)}
$$

The calculation using the formula above shows that the minimum sample for each group is 40 caregivers. This calculation is based on $Z \alpha$ distribution $=1.96 ; Z \beta$ distribution $=0.84$; standard deviation $(\sigma)$ caregiver burden $=6.4$; and size of effect prediction $=4$. We add 2 samples to each group to anticipate sample dropout or loss of follow-up. There were 2 samples in each group dropout during this study. The number of samples completed in the study was 40 samples for each group. All samples in this research gave written informed consent.

\subsection{Procedures}

\subsubsection{The CEP-BAM}

The intervention group in this study received the CEPBAM, the interventions to empower family caregivers so that they can care and facilitate patients undergoing post-stroke adaptation at home. The interventions include providing information to caregivers about stroke and prevention of stroke recurrent, training the caregiver to apply adaptive coping and physical and psychosocial adaptation, and teaching them how to maintain adequate psychological conditions when caring for the patient. These interventions aim to reduce the burden of family caregivers and improve the quality of life of patients. We empowered the caregiver to be able to help the patient undergo the process of post-stroke adaptation to achieve optimal quality of life after a stroke. CEP-BAM also trains caregivers on how to deal with patient problems, and improve coping behavior and adaptation responses of patients. This intervention is evidence-based to improve health outcomes in poststroke patients. ${ }^{10}$

There are 3 stages of CEP-BAM interventions, including pre-education, intervention, and monitoringevaluation. We take a psychological approach and build trusting relationships with caregivers in the preeducational stage. Then in the second stage, we carried out 6 interventions in 2 weeks; interventions 1 to 3 were carried out in the first week and interventions 4 to 6 were carried out in the second week. In intervention 1 we provided caregivers with information about stroke, how to prevent recurrent strokes, and problems experienced by patients. In intervention 2 we trained caregivers to carry out post-stroke adaptive coping strategies. In intervention 3 we trained caregivers to carry out poststroke physical adaptation (ambulation). In intervention 4 we trained caregivers to carry out walking and joint movement exercises. In intervention 5 we trained caregivers to carry out daily activities including bathing, toileting, dressing, and feeding exercises for stroke patients. In intervention 6 we taught caregivers about emotional support to patients and how to maintain adequate psychological health while caring for patients. In the third stage, we reinforce caregivers and conduct monitoringevaluation. This last stage is carried out from the third week to the last week of the sixth month. We monitored patient progress, caregiver's ability to care for patients, and caregiver's burden. We motivated caregivers, give 
them support, help them solve problems, and strengthen their positive achievements. ${ }^{10}$ We evaluated caregiver's coping and their burden in caring for patients at the end of the sixth month.

\subsubsection{Control}

The control group received a conventional intervention in the form of a discharge planning program for family caregivers in the hospital. The discharge planning program consists of an assessment of caregiver information needs, education about stroke and post-stroke care at home, education about the physical and psycho-social recovery process after stroke, and how to carry out the role of caregiver. The nurses in the hospital followed up, monitored, and reinforced the caregivers via telephone 1 time in 1 week for 6 months. We evaluated caregiver's coping and their burden in caring for patients at the end of the sixth month.

\subsection{Data collection}

We conducted this study for 8 months from February to September 2017, including intervention, monitoring, follow-up, and data collection. We used 4 observers who were trained, understood measurement methods, and had made the same perception among observers in this study. Measurements in this study used the principle of single-blind, in which observers do not know into which group the sample is allocated. The principle of single-blind measurement is to ensure the validity of research results.

The instrument used in this study consisted of a caregiver coping questionnaire to measure coping strategies of caregivers, caregiver burden scale (CBS) to measure caregiver burden in caring for stroke patients, and Barthel's Index (BI) to determine the category of patient basic activity assistance. ${ }^{11}$ The CBS psychometric test showed satisfactory results and hence this instrument was found suitable for use in measurement. Confirmatory factor analysis shows that all CBS dimensions have internal consistency between 0.698 and 0.755 . Instrument reliability testing using test-retest showed results in reliability between 0.745 and 0.900 . When compared with similar instruments, all CBS dimensions are significantly correlated with the Beck Depression Inventory (BDI) and the World Health Organization Quality of Life (WHO-QoL).12 CBS has good validity and reliability to measure the burden of caregivers. ${ }^{13}$

\subsection{Data analysis}

Data analysis in this study was conducted using bivariate and multivariate analysis. Bivariate analysis was performed to examine the homogeneity of caregiver characteristics between the two groups. We analyzed the differences in caregiver characteristics between the two groups on a categorical scale such as education level, caregiver-patient relationship, sex, type of stroke, and level of patient basic activity assistance using the $X^{2}$ test, whereas differences in age of caregivers between groups were analyzed using an independent $t$ test. The multivariate analysis in this study used the analysis of variance (ANOVA) repeated measure to determine the difference in caregiver's coping and burden between the 4 measurements between the 2 groups after being controlled by confounding variables.

\section{Results}

There are 80 caregivers who completed this study. Figure 1 shows the research flow diagram.

Table 1 shows most caregivers in both groups were high school graduates $(47.5 \%$ in the intervention group and $67.5 \%$ in the control group), the patient's wife/ husband $(37.5 \%$ in the intervention group and $45 \%$ in the control group), female $(67.5 \%$ in the intervention group and $72.5 \%$ in the control group), and give the basic activity assistance for patients in the level of moderate $(62.5 \%$ in the intervention group and $57.5 \%$ in the control group). Based on the stroke type most of the patients are non-hemorrhagic $(87.5 \%$ in the intervention group and $82.5 \%$ in the control group). The mean age of caregivers was 43.38 years in the intervention group and 41.13 years in the control group. The equality test of caregiver characteristics between the two groups showed no significant difference in all variables $(P>0.050)$.

Table 2 shows differences in the caregiver's coping between measurements between the two groups after being controlled by confounding variables (stroke type, basic activity assistance provided by the caregiver, age, caregiver-patient relationship, and education level). We found the different trends in caregiver's coping after intervention in both groups. Intervention groups showed consistency in the increase of coping skills from posttest 1 to post-test 3, while the control group showed fluctuating changes that decreased in post-test 1 and then increased in post-test 2 and post-test 3 . Bivariate analysis showed there were no significant differences in caregiver's coping in the pre-test and post-test 1 between the two groups $(P>0.050)$. Significant differences in caregiver's coping between the two groups occurred in the post-test-2 and post-test 3 , in which caregiver's coping in the intervention group was better than the control group $(P<0.050)$. Multivariate analysis using a general linear model repeated measure showed significant 


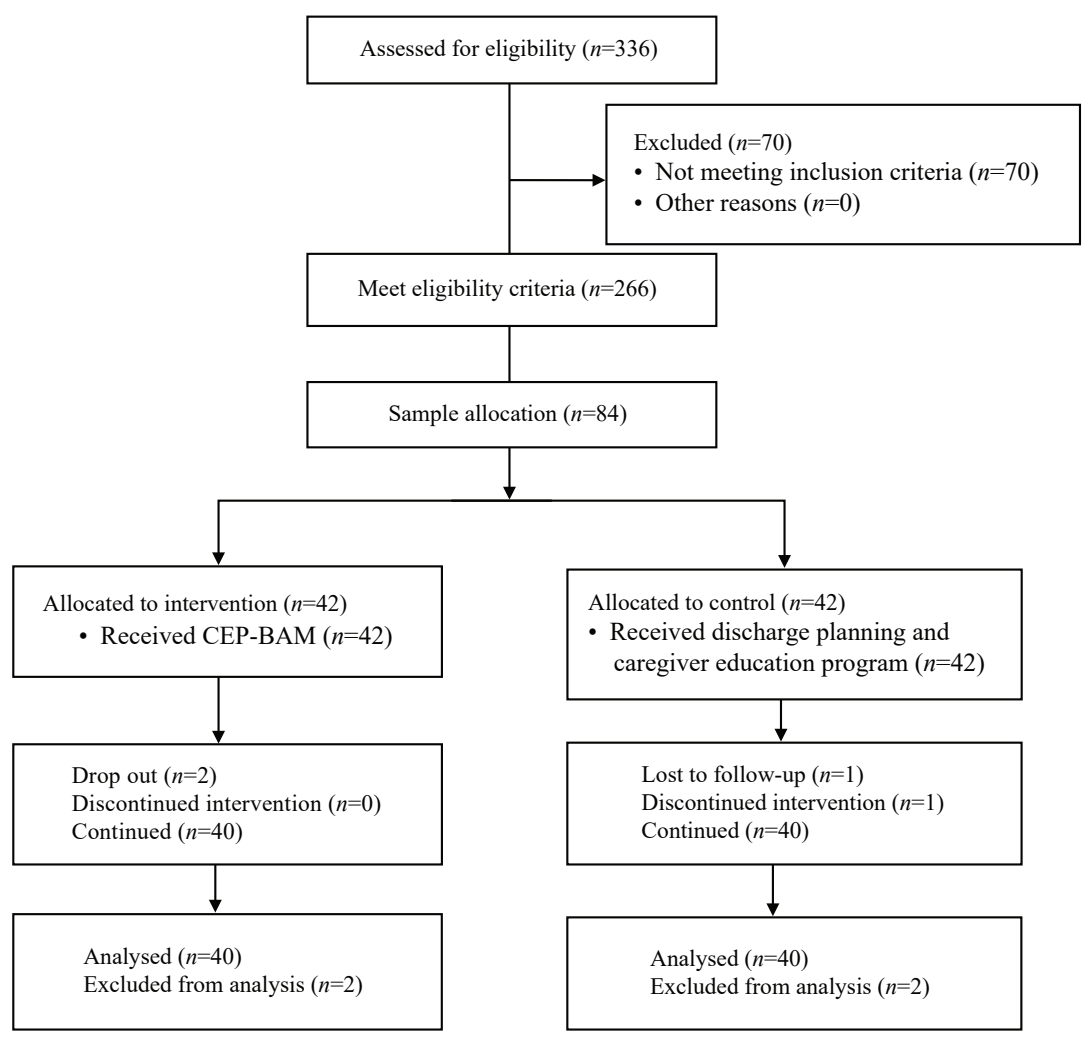

Figure 1. Research flow diagram. CEP-BAM, Caregiver Empowerment Program Based on the Adaptation Model.

\begin{tabular}{|c|c|c|c|c|c|c|}
\hline \multirow{2}{*}{ Variables } & \multicolumn{2}{|c|}{ Intervention } & \multicolumn{2}{|c|}{ Control } & \multirow{2}{*}{$\chi^{2}$ or $t$ value } & \multirow{2}{*}{$P$ value } \\
\hline & $n$ & $\%$ & $n$ & $\%$ & & \\
\hline Education level & & & & & 4.269 & 0.234 \\
\hline Undergraduate & 6 & 15 & 8 & 20 & & \\
\hline Senior high school & 19 & 47.5 & 25 & 62.5 & & \\
\hline Junior high school & 10 & 25 & 4 & 10 & & \\
\hline Elementary school & 5 & 12.5 & 3 & 7.5 & & \\
\hline Caregiver-patient relationship & & & & & 1.152 & 0.765 \\
\hline Husband/wife & 15 & 37.5 & 18 & 45 & & \\
\hline Son/daughter & 13 & 32.5 & 14 & 35 & & \\
\hline Son in law/daughter in law & 8 & 20 & 5 & 12.5 & & \\
\hline Sibling & 4 & 10 & 3 & 7.5 & & \\
\hline Sex & & & & & 0.060 & 0.807 \\
\hline Male & 13 & 32.5 & 11 & 27.5 & & \\
\hline Female & 27 & 67.5 & 29 & 72.5 & & \\
\hline Stroke type & & & & & 0.098 & 0.754 \\
\hline Non-hemorrhagic & 35 & 87.5 & 33 & 82.5 & & \\
\hline Hemorrhagic & 5 & 12.5 & 7 & 17.5 & & \\
\hline Basic activity assistance provided by the caregiver & & & & & 0.052 & 0.819 \\
\hline Minimal & 15 & 37.5 & 17 & 42.5 & & \\
\hline Moderate & 25 & 62.5 & 23 & 57.5 & & \\
\hline Caregiver's age (Mean, SD) & 43.38 & 11.34 & 41.13 & 11.50 & 0.881 & 0.381 \\
\hline
\end{tabular}

Table 1. The characteristics of caregivers $(n=80)$ 


\begin{tabular}{|c|c|c|c|c|c|c|}
\hline \multirow{2}{*}{ Measurement } & \multicolumn{2}{|c|}{ Intervention $(n=40)$} & \multicolumn{2}{|c|}{ Control $(n=40)$} & \multirow{2}{*}{$t$ value } & \multirow{2}{*}{$P$ value } \\
\hline & Mean & SD & Mean & SD & & \\
\hline Pre-test & 114.35 & 9.58 & 113 & 9.98 & 0.617 & 0.593 \\
\hline Post-test 1 & 116.13 & 9.56 & 112.93 & 11.60 & 1.346 & 0.182 \\
\hline Post-test 2 & 117.50 & 9.73 & 113.10 & 10.13 & 1.981 & 0.051 \\
\hline Post-test 3 & 119.65 & 10.36 & 113.90 & 10.13 & 2.510 & 0.014 \\
\hline
\end{tabular}

Note: The difference between pre-test and post-test 1 between groups $(P=0.100)$; the difference between pre-test and post-test 2 between groups $(P=0.026)$; the difference between pre-test and post-test 3 between groups $(P=0.009)$.

Table 2. The caregiver's coping between measurements in both groups $(n=80)$.

\begin{tabular}{lcccccc}
\hline \multirow{2}{*}{ Measurement } & \multicolumn{2}{c}{ Intervention $(\mathrm{n}=40)$} & \multicolumn{2}{c}{ Control $(n=40)$} & & \multirow{2}{*}{ P value } \\
\cline { 2 - 4 } & Mean & SD & Mean & SD & & 0.494 \\
\hline \hline Pre-test & 35.80 & 5.44 & 34.95 & 5.62 & 0.687 & 0.264 \\
Post-test 1 & 33.75 & 6.02 & 35.20 & 5.48 & -1.126 & 0.297 \\
Post-test 2 & 33.33 & 5.95 & 34.78 & 6.38 & -1.051 & 0.162 \\
Post-test 3 & 33.00 & 4.86 & 34.70 & 5.85 & -1.413 & 0.13 \\
\hline
\end{tabular}

Note: The difference between pre-test and post-test 1 between groups $(P=0.001)$; the difference between pre-test and post-test 2 between groups $(P=0.006)$; the difference between pre-test and post-test 3 between groups $(P=0.014)$.

Table 3. The caregiver's burden between measurements in both groups $(n=80)$.

differences in caregiver's coping between measurements between the two groups $(P=0.016)$. Significant differences in caregiver's coping between the two groups occurred between pre-test and post-test $2(P=0.026)$ and between pre-test and post-test $3(P=0.009)$. This analysis shows that CEP-BAM is effective in increasing caregiver's coping in post-test 2 (5 months after intervention) and continues until post-test 3 (6 months after intervention).

Table 3 shows differences in the caregiver's burden between measurements between the two groups after being controlled by confounding variables (stroke type, basic activity assistance provided by the caregiver, age, caregiver-patient relationship, and education level). We found the different trends in caregiver's burden after intervention in both groups. Intervention groups showed consistency in the decrease of caregiver burden from post-test 1 to post-test 3, while the control group showed fluctuating changes that increased in post-test 1 and then decreased in post-test 2 and post-test 3 . Multivariate analysis showed significant differences in the caregiver's burden between measurements between the two groups $(P=0.009)$. Significant differences were found between pre-test and post-tests with the biggest difference between pre-test and post-test $1(P=0.001)$. This analysis showed that CEP-BAM was effective in reducing the burden of caregivers starting from post-test 1 (4 months after intervention) and continued until posttest 3 (6 months after the intervention).

\section{Discussion}

\subsection{CEP-BAM improves stroke caregiver's coping}

This research proved that CEP-BAM is effective in improving the coping strategies of the caregiver in dealing with problems while caring for stroke patients. CEP-BAM interventions have been shown to increase the ability of caregivers in using adaptive coping strategies. The caregiver is not only taught how to care for post-stroke patients at home but is also trained to use adaptive coping strategies when dealing with problems. These coping strategies can reduce the burden of family caregivers, prevent stress, and maintain their quality of life in the optimal range. The use of active coping 
strategies is a predictor for achieving a good quality of life in family caregivers who care for stroke patients. ${ }^{14}$ This study recommends the need for community nurses to help and teach family caregivers how to deal with their problems, use active coping, and seek instrumental and emotional support when experiencing problems in caring for stroke patients at home.

We empower caregivers to use adaptive psychological coping including positive thinking, accepting current conditions, and controlling emotions. We instill awareness to caregivers that most of them experience the same problem even more severely, and many of them can adapt to dealing with these problems. We also assure caregivers that they can provide quality care that will have an impact on accelerating patient recovery. Positive thinking can increase caregiver's confidence in their ability to care for patients. We teach caregivers how to maintain a healthy psychological condition while caring for stroke patients. This method can help caregivers in reducing stress and burden. We also teach caregivers to apply religious-spiritual coping, which is a method of self-defense by getting closer to God, accepting all the decrees of God, and believing that God will provide the best solution for all the problems they experience. Caregivers who apply this coping will accept the current conditions, have high motivation, and have the confidence to care for patients.

We teach caregivers how to control emotions such as being patient and not getting angry when facing problems, not dragging on thinking about problems, and thinking about something that makes the mind happy. Making an effort not to dissolve in sadness and not to think about the problem as severe will increase caregivers' selfacceptance of the conditions they experience. Caregivers who accept the reality of current conditions will not experience stress and will have a good self-concept, so they can adjust more positively as time goes by. The use of new ways of doing activities also contributes greatly to the process of caregivers accepting and adjusting to their roles.

Caregivers who perceive a heavy burden in caring for stroke patients can experience depressive symptoms. A study showed that factors related to depression in caregivers included unclear post-stroke care, stress, social support, income, time spent caring for patients, coping strategy, and functional capacity of the patient. ${ }^{15}$ Coping strategy in dealing with problems when caring for the patient largely determines the intensity of the burden felt by caregivers, which in turn affects the level of depression they experience. Nurses need to train caregivers to reduce their anxiety and burden by applying adaptive coping strategies including problem-solving methods, positive self-assessment, assertive behavior, and curtailing negative thinking. Caregivers who use adaptive coping strategies will show a low burden and a high quality of life. Research shows that social support for caregivers and the use of active coping strategies can improve their quality of life.$^{14}$ Interventions are needed to increase social support for caregivers and improve their coping strategies such as implementing active coping, seeking instrumental and emotional support, and changing negative thoughts such as denying and blaming themselves. ${ }^{14}$

\subsection{CEP-BAM decrease stroke caregiver burden}

This study proved that CEP-BAM was an effective intervention to reduce the burden of caregivers starting from 4 months and continuing until 6 months after the intervention. CEP-BAM is a collection of interventions to empower family caregivers to be able to adapt when caring for stroke patients at home. CEP-BAM provides caregivers with information about stroke and post-stroke care to increase their awareness of problems due to stroke and improve their skills to care for the patient. A good understanding of stroke and post-stroke care at home contributes to reducing the caregiver burden. Adequate caregivers' knowledge and skills in caring for stroke patients will increase their confidence, giving rise to a positive perception that caring for patients is not a burden. The daily time to care for patients is related to caregiver burden..$^{16}$ In this regard, the nurse needs to develop and provide home health care for post-stroke patients and their caregivers to improve patient outcomes and decrease caregivers' burden.

Most caregivers feel the burden of caring for stroke patients consistently over time. ${ }^{17}$ The burden of caring for stroke patients has an impact on the physical and psychological health of caregivers. They may experience fatigue, anxiety, and depression. This problem will have an impact on their ability to care for stroke patients at home. Management in post-stroke patients will not achieve maximum results if it does not integrate caregiver interventions. ${ }^{18}$ The nurse needs to help and empower caregivers so that they can adapt effectively when facing various problems in caring for patients. Caregiver empowerment includes several interventions to improve their knowledge and skills in caring for stroke patients at home, improve coping strategies, and improve adaptive behavior in dealing with stressors.

Caregivers who feel a heavy burden while caring for patients tend to experience a decrease in productivity and quality of life. The burden experienced by caregivers also causes a decrease in the quality of care they provide to patients, which can prolong recovery time and reduce the quality of life of patients. Based on this, interventions for post-stroke patients need to integrate interventions to reduce the burden of family caregivers. 
Family caregiver health status, length of stay in the hospital, and time needed to care for the patient per day affect the quality of life of a family caregiver, with the burden of the family caregiver as a mediator variable. ${ }^{19}$ The study above concludes that nurses need to train caregivers to manage their time effectively in caring for patients and teach them how to maintain their physical and psychological health status while caring for patients. CEPBAM interventions include educating caregivers about how to adapt while caring for the patient, how to care for patients at home, and how to maintain a healthy physical and psychological condition while caring for patients. Through this intervention, caregivers are expected to be able to provide quality care to patients using effective time, so they still have time for other activities. Optimal health conditions and the availability of free time to do other activities besides caring for patients will reduce the burden experienced by caregivers.

Information provided by nurses to family caregivers during discharge planning in hospitals is not sufficient to improve patient outcomes and reduce their burden. They need to get more support than just information during discharge planning at the hospital. Nurses need to empower family caregivers comprehensively, including interventions to improve their knowledge and skills about stroke care at home, adaptive coping strategies, and problem-solving methods. Research shows that the intervention to family caregivers, which are only in the form of education without comprehensive empowerment, do not show significant results in improving the functional abilities of patients. ${ }^{20}$ The nurse needs to develop new interventions in rehabilitation for post-stroke patients such as home visits to empower family caregivers. They need special attention and guidance so that they can adapt to various stressors and show adequate skills in caring for stroke patients at home.

The CEP-BAM teaches caregivers about adaptive coping strategies in dealing with problems when treating patients at home. Empowerment of family caregivers by improving coping strategies will have an impact on increasing their ability to deal with problems, increasing live management skills, and increasing confidence in problem-solving. This will have a positive impact not only on caregivers but also on post-stroke patients. The benefits for caregivers are reduction in the burden they experience while treating patients, while the benefits for patients are enhancement of functional capacity and quality of life through quality care and assistance by caregivers. The study by Lui, Lee, Greenwood, and Ross (2011) reinforces the fact that the ability of family caregivers to solve their problems and self-confidence 1 week before discharge from the hospital is related to the physical health and social support they feel after 3 months post-discharge. ${ }^{21}$ This indicates that maximizing the confidence of caregivers and increasing their ability to solve problems will be very valuable for them in caring for stroke patients. Collaboration between nurses and caregivers when discharged from the hospital and the initial transition at home will provide great support to caregivers in preparing themselves to care for patients at home. ${ }^{21}$

It is necessary to develop stroke health care services at home to improve the health outcomes of stroke patients and their caregivers. This study proved a consistent decrease of caregiver burden from posttest 1 to post-test 3 after CEP-BAM in the intervention group. The decrease in post-test 2 and 3 was not as great as in post-test 1 . Based on these findings, there was an opportunity to reduce the caregiver's burden 1 month after the intervention through adequate support to them. The nurse needs to reinforce intense support and monitoring when entering the second month to maintain the trend of decreasing the caregiver's burden on the linear line.

In addition, we also evaluate the use of supporting tools including modules and booklets. We asked caregivers about the modules and booklets based on aspects of language, their ability to understand the contents of the modules, and the ability to implement the recommendations in the modules. We made a revision based on the caregiver's opinion about the contents of the modules. Revisions were made to simplify sentences using language that was easily understood by caregivers with various levels of education. The limitation of this study is not identifying the factors that mediate the effect of CEP-BAM on caregiver's burdens such as problemsolving and adherence to follow the guideline provided by the nurse.

\section{Conclusion}

The CEP-BAM interventions are effective in increasing adaptive coping strategies and reducing the burden of caregivers 4 months and continuing 6 months after the intervention.

\section{Acknowledgments}

We express our deepest gratitude to the observers who have assisted in the collection of data, the family caregivers who were willing to be sampled, the medical records officers of regional general hospitals in West Kalimantan and Jakarta who have facilitated us in searching for sample data, and the Board for Development and Empowerment Human Resources of Health, 
the Indonesian Ministry of Health who have been funded for this research.

\section{Ethics approval}

In order to guarantee the confidentiality of the research subject's identity, uphold human dignity and rights, and ensure that the benefits derived from this study outweigh the negative impacts, the research protocol has been through a careful review process by the research ethics committee of Faculty of Medicine Tanjung Pura University with Institutional Review Board (No. 5321/ UN22.9/DT/2016).

\section{Conflicts of interest}

None of the authors disclose any potential conflict of interest.

\section{References}

1. Loh AZ, Tan JS, Zhang MW, Ho RC. The global prevalence of anxiety and depressive symptoms among caregivers of stroke survivors. J Am Med Dir Assoc. 2017;18:111-116.

2. Menon B, Salini P, Habeeba K, Conjeevaram J, Munisusmitha K. Female caregivers and stroke severity determines caregiver stress in stroke patients. Ann Indian Acad Neurol. 2017;20:418-424.

3. Hu P, Yang Q, Kong L, Hu L, Zeng L. Relationship between the anxiety/depression and care burden of the major caregiver of stroke patients. Medicine (United States). 2018;97:e12638.

4. Caro CC, Mendes PVB, Costa JD, Nock LJ, da Cruz DMC. Independence and cognition post-stroke and its relationship to burden and quality of life of family caregivers. Top Stroke Rehabil. 2017;24:194-199.

5. Peng Y, Brown CC, Zhang J, et al. An examination of a simplified stroke rehabilitation program for reducing family caregiver's burden for stroke patients in rural China. Contemp Fam Ther. 2019;41:168-179.

6. Pitthayapong $S$, Thiangtam W, Powwattana A, Leelacharas S, Waters CM. A community based program for family caregivers for post stroke survivors in Thailand. Asian Nurs Res (Korean Soc Nurs Sci). 2017;11:150-157.

7. Cheng HY, Chair SY, Chau JPC. Effectiveness of a strength-oriented psychoeducation on caregiving competence, problem-solving abilities, psychosocial outcomes and physical health among family caregiver of stroke survivors: a randomised controlled trial. Int J Nurs Stud. 2018;87:84-93.
8. Deyhoul N, Vasli P, Rohani C, Shakeri N, Hosseini M. The effect of family-centered empowerment program on the family caregiver burden and the activities of daily living of Iranian patients with stroke: a randomized controlled trial study. Aging Clin Exp Res. 2020;32:1343-1352.

9. Chang AK, Park Y-H, Fritschi C, Kim MJ. A family involvement and patient-tailored health management program in elderly Korean stroke patients' day care centers. Rehabil Nurs. 2015;40:179-187.

10. Dharma KK, Damhudi D, Yardes N, Haeriyanto S. Increase in the functional capacity and quality of life among stroke patients by family caregiver empowerment program based on adaptation model. Int $J$ Nurs Sci. 2018;5:357-364.

11. González N, Bilbao A, Forjaz MJ, et al. Psychometric characteristics of the spanish version of the barthel index. Aging Clin Exp Res. 2018;30: 489-497.

12. Farajzadeh A, Akbarfahimi M, Maroufizadeh $S$, Rostami HR, Kohan AH. Psychometric properties of Persian version of the Caregiver Burden Scale in Iranian caregivers of patients with spinal cord injury. Disabil Rehabil. 2018;40:367-372.

13. Lee K-C, Yiin J-J, Chao Y-F. Development of an experience-based Caregiver Burden Scale in advanced cancer. Cancer Nurs. 2015;39:12-19.

14. Yu Y, Hu J, Efird JT, Mccoy TP. Social support, coping strategies and health-related quality of life among primary caregivers of stroke survivors in China. J Clin Nurs. 2013;22:2160-2171.

15. Byun E, Evans L, Sommers M, Tkacs N, Riegel B. Depressive symptoms in caregivers immediately after stroke. Top Stroke Rehabil. 2019;26:187-194.

16. Kamel AA, Bond AE, Froelicher ES. Depression and caregiver burden experienced by caregivers of Jordanian patients with stroke. Int $\mathrm{J}$ Nurs Pract. 2012;18:147-154.

17. Pont W, Groeneveld I, Arwert H, et al. Caregiver burden after stroke: changes over time? Disabil Rehabil. 2020;42:360-367.

18. O'Shea R, Goode D. Effects of stroke on informal carers. Nurs Stand. 2013;28:43-47.

19. Jeong YG, Jeong YJ, Kim WC, Kim JS. The mediating effect of caregiver burden on the caregivers' quality of life. J Phys Ther Sci. 2015;27:1543-1547.

20. Mudzi W, Stewart A, Musenge E. Effect of carer education on functional abilities of patients with stroke. Int J Ther Rehabil. 2012;19:380-385.

21. Lui MHL, Lee DTF, Greenwood N, Ross FM. Informal stroke caregivers' self-appraised problem-solving abilities as a predictor of well-being and perceived social support. J Clin Nurs. 2012;21:232-242. 